\section{Memory: Episodic}

Yee Lee Shing

Center for Lifespan Psychology, Max Planck

Institute for Human Development, Berlin,

Germany

\section{Synonyms}

Declarative memory; Explicit memory; Long-term memory

\section{Definition}

Episodic memory refers to memory about events that are bound to particular times and places in the past. It is a type of long-term memory containing information from the past (ranging from minutes to decades ago) that can be recalled explicitly (hence also called explicit or declarative memory). A core part of our identity is rooted in the ability to mentally travel back in time and reexperience past events. For example, to remember what happened on your last birthday is episodic memory.

Episodic remembering is a conscious retrieval process, such that an individual attempts to reconstruct the past personal experience. It can be accompanied by specific contextual details of the episode, generally referred as recollection, or only based on the feeling of remembering without recovering specific details, generally referred as familiarity. While most of the time retrieval of past experience can be achieved reliably, the adaptive nature of the human memory system also renders itself prone to errors and distortions.

\section{Introduction}

Most people associate aging with declines in episodic memory. Older adults more often complain about experiencing memory difficulties, such as learning new information or recalling the name of a familiar face. Empirical evidence lends some support to this common conception. Crosssectional studies, which involve a study sample consisting of individuals of different chronological age, suggest that individuals who are older show lower memory performance than those who are younger (Kausler 1994). Linear deterioration in episodic memory performance begins as early as in the $20 \mathrm{~s}$. Longitudinal and cohortsequential studies in adulthood, which follow one or a few groups of individuals repeatedly across years, also show that episodic memory declines across time in old age. However, an important divergence in finding emerged such that episodic memory performance seems to remain relatively stable until about $60-65$ years of age, after which accelerating decline is observed (Nyberget al. 2012). One source of discrepancy in estimating the onset of decline may be because cross-sectional estimates of age-related 
difference are potentially biased by cohort effects, with later generations showing higher educational attainment and better memory performance. In any case, it is fair to say that the decline in episodic memory is reliably observed in old age (particularly after age 60) and is steeper than semantic memory, namely declarative information about the world, culture, and one's own environment. This is the case even in individuals who are healthy and show no signs of dementing illness. A number of studies have examined cognitive decline in relation to proximity to death in older individuals (i.e., terminal decline). Accelerated decline in episodic memory has been identified as early as 8.4 years prior to death, with a rate twice that observed as a function of chronological age. At the same time, the structure, function, and neurochemistry of brain circuitries supporting episodic memory also undergo profound reorganization in old age (Buckner 2004). Due to the increased availability of brain-imaging technology, one of the current focuses in the aging research fields is to better understand the links and dependencies between cognitive and neural changes during late life.

\section{Differential Age-Related Differences in Memory Processes}

There are a number of important qualifications to the mean declining trend in episodic memory. The extent of episodic memory decline varies with the type of memory processes entailed. In the laboratory, episodic memory is typically studied by presenting the participants with some to-beremembered information (e.g., encoding a list of scene pictures or word pairs). After a retention interval (ranging from minutes, days, to weeks), participants' memory on the information is tested or retrieved. One commonly used test format is recognition, in which studied and non-studied items are presented and participants' task is to say "old" to the studied items and "new" to the non-studied items. Another commonly used test format is cued recall or recall, in which participants have to search through their memory actively (or based on a retrieval cue) to remember the studied items, monitor the retrieved information, and judge whether it is the appropriate target given the current retrieval context.

Different aspects of memory performance are disproportionally affected by aging. In general, the extent of age differences in episodic memory depends on the amount of self-initiated processing required by the task. According to Craik (1983), older adults' memory performance is particularly impaired when retrieval depends on self-guided cues and active control processes. This is due to age-related depletion of attentional resources of the mind. On the other end of the continuum, with more task-appropriate cue from the environment, age-related deficits should be reduced (cf. Lindenberger and Mayr 2014). Empirical support for these propositions can be found in the literature. For example, older adults are less effective than younger adults in using memory strategies to aid remembrance. Age differences tend to be smaller by increasing schematic support afforded by the study material. When memory tasks involve naturalistic information where participants can make use of prior knowledge, age-related differences can be reduced or eliminated. Examples for this include studying grocery items with price information at market value or word pairs comprising words semantically related to each other. The same phenomenon is found for retrieval from episodic memory. Processing demands at retrieval are greater in recall than recognition due to the additional needs to search and monitor the retrieval process. In line with this, age differences found in recall tasks are typically larger than with recognition tasks.

In addition to the central aspects of information, episodic memory heavily involves the encoding and retrieval of contextual information surrounding the episode, such as the modality in which information was presented or spatial location of items. It has been shown that memory for content shows smaller age-related decrease than memory for context (Spencer and Raz 1995). The magnitude of age differences in context memory depends on the relevance of the context to the targeted content. In a more general form of the content-context distinction, Chalfonte and Johnson (1996) proposed that older adults have 
difficulties binding pieces of information into complex memories. Later on, this was formulated as the associative deficit hypothesis, which postulates that older adults' problems in episodic memory are due to their difficulties in creating and retrieving intra- und inter-item associations. Empirical evidence has been gathered for this hypothesis, indicating a larger effect of age on memory for associative than for item information (Old and Naveh-Benjamin 2008). This was found in memory for source, context, temporal order, spatial location, and item pairings, in both verbal and nonverbal material, and particularly under intentional learning instructions. Additionally, age-related deficits in associative memory, particularly when measured with recognition, are due to higher false-alarm rate in wrongly endorsing rearranged information as original studied material (Shing et al. 2010).

One explanation for such a pattern of results is based on the dual process account of recognition memory (Yonelinas 2002). It is suggested that memory for past events can be based on retrieval accompanied by specific contextual details, that is, recollection, or on the feeling that an event is old without recovering specific details, i.e., familiarity. It is shown that recollection of particular contextual details is more strongly affected by senescent changes than familiarity-based processing (Light et al. 2000). Older adults are also more susceptible to familiarity-based memory illusions, such as ironic effects of repetition, in which repeated lures (unstudied information) during a recognition memory test are mistaken for word originally presented during encoding. Taken together, older adults show a stronger reliance on familiarity feeling during recognition, leading them to be more readily to falsely endorse information that appears familiar without engaging in careful verification through cognitive control.

\section{False Memories in Aging}

In older adults, the reduction in memory for context, and the increased reliance on general familiarity, makes them particularly vulnerable for committing false memory (Schacter et al. 1997;
Jacoby and Rhodes 2006). It has been shown that older adults, in addition to worse veridical memory, exhibit higher tendency to falsely remember things that in fact did not take place in the past. False memory is commonly studied using the Deese-Roediger-McDermott paradigm. Here, participants study a list of words in which some of them are related to a common theme (e.g., pillow, dream, and rest are related to sleep). Older adults show a more pronounced increase than younger adults in the erroneous endorsing or recall of critical lures (i.e., sleep) after studying the semantically related items in the encoding list. Furthermore, manipulations that reduce false memories in younger adults, such as providing more encoding opportunities or warning about the semantic lures, are only partially effective in older adults. For example, after several repeated study-test trials in which the same lists were presented, only younger but not older adults showed a decrease in recalling non-presented semantic lures. This showed that older adults have difficulties in building up veridical representations of the actually studied items. At the same time, as both studied and non-studied semantic lures become more familiar across repetitions, older adults are less able to control their decision against the increasing strength of familiarity signals. Research also shows that older adults have difficulties in remembering the source of information, such as whether an event was suggested or perceived, who presented the information, or if the participant asked for, answered, or listened to certain information. Furthermore, older adults' subjective experience of memory (in the form of self-reported confidence judgment) is less well calibrated to their actual level of accuracy. In some cases older adults have been found to be falsely confident about the accuracy of their recognition memory (Shing et al. 2010). This renders older adults more susceptible to falsely endorsing misleading information (the misinformation effect) and scams in real life. 


\section{Interindividual Difference in Memory Aging}

Despite the general mean trend of memory decline, there are vast amounts of interindividual differences in both level and change of memory performance in late life. This suggests that while some individuals experience massive deterioration in memory functioning, other highperforming individuals display little or no performance decline in memory (Nyberg et al. 2012). These interindividual differences in change of memory increase as a function of advancing adult age.

Basic cognitive resources including perceptual speed, working memory, and inhibition are reported to be important predictors of interindividual differences in memory performance. This was commonly studied by correlational methods testing for mediation, such that the resource factors are measured independently and their roles in accounting for cross-sectional age-related variance in memory performance are determined statistically. Skepticism is warranted against such approach as it is limited in its ability to explicate within-person longitudinal changes accurately (Lindenberger et al. 2011). To understand interindividual differences in level and change of memory functioning, researchers need to rely on longitudinal investigations of memory development within individuals.

Factors related to lifetime exposures such as educational attainment, socioeconomic status, and occupation have been considered as moderators of the age-memory relationship. This line of work is related to the notion of cognitive reserve, which postulates that cognitive processes are crucial for explaining the differences between someone who is functionally impaired and someone who is not, despite equal brain changes or pathology (Barulli and Stern 2013). These cognitive processes reflect differences in cognitive efficiency presumably shaped by life experiences. Empirically it is often estimated using proxy variables for lifetime exposures and cognitive activity, such as educational attainment. However, existing data suggests that education is associated with level of memory performance in old age, but not with interindividual differences in change, likely reflecting people's differences in cognitive functioning accumulating since early life. On the other hand, data from longitudinal observational studies suggests that having an enriched lifestyle that is intellectually stimulating and physically active predicts better maintenance of cognitive skills and is associated with a reduced risk of developing Alzheimer's disease in late life (Hertzog et al. 2009). Having an occupation of higher complexity in life is related to higher cognitive performance, but this relationship is attenuated after retirement, suggesting that forming as well as maintaining a stimulating lifestyle is important for preserving cognitive functioning in late life (Nyberg et al. 2012). Note that caution is warranted given that causal directionality of the association between enriched experience and cognitive functioning in human is thus far difficult to disentangle.

\section{Plasticity in Memory Aging}

It is assumed that aging is not consisting of fixed trajectories but rather a range of possible functioning levels, which reflects person-specific constellations ranging from genetic profile to lifestyle preference. Given the observed memory decline in aging, this begs the question of to what extent (and in which condition) there is remaining memory plasticity in cognitively healthy older adults (Hertzog et al. 2009). Earlier memory training research focused on instructing older adults with memory strategy, as they are less able to spontaneously initiate adequate encoding strategies (Kausler 1994). Instruction and/or practice in a memory encoding technique, for example using imagery strategy, leads to robust performance improvements in healthy older adults. However, thus far the effects of strategy-based training seem to be relatively constrained to the memory task being trained and lack transfer to other cognitive domain or real-life application.

Reducing false memory in older adults can partly be achieved by training retrieval processes. After training in situations that entail facing high levels of interference and being provided by 
feedback, older adults can increase reliance on recollection and reduce misleading automatic influences of memory, such as the familiarity signal (Jacoby and Rhodes 2006). Such kinds of recollection training show transfer to other classes of memory tasks that are prone to proactive interference and require weighing of competing information.

Aerobic exercise as an intervention paradigm has better documented benefits in improving performance on cognitive tests, including episodic memory (Smith et al. 2010). It has been shown that aerobic exercise training in older adults increased the size of the anterior hippocampus, reversing the amount of loss by 1-2 years. The gain was also related to improvements in spatial memory. The neural mechanisms driving the positive effects of aerobic fitness on brain status and cognitive performance in humans are thus far not well understood. Evidence from animal studies suggests that these effects may be associated with neurogenesis in the hippocampus, and the dentate gyrus in particular, due to changes in neurotransmitter and growth factor release (Kempermann 2008).

\section{Aging Brain and Memory}

In recent years, many studies have used magnetic resonance imaging to examine the neural correlates of episodic memory decline in aging. Age-related differences and in some cases longitudinal decline in the structural and functional integrity of the neural network supporting episodic memory, particularly the prefrontal cortex and medial-temporal lobe, are frequently observed. Prefrontal brain regions are among the areas that undergo the strongest atrophy in old age (Raz 2005). Gray matter changes are especially pronounced in the hippocampus and less so in the surrounding cortex in the medial-temporal lobe. Hippocampal atrophy is particularly visible in those individuals with hypertension, underscoring the importance of vascular health in aging.

Studies assessing age-related differences in functional activation have mostly used the subsequent memory paradigm, in which encoding activity related to subsequently remembered events is contrasted against activity related to subsequently forgotten ones. In a meta-analysis by Maillet and Rajah (2014), age-related decreases in subsequent memory effect in the occipital and fusiform cortex are observed. But older adults tend to over recruit in a set of regions including bilateral middle/superior frontal gyri, anterior medial frontal gyrus, precuneus, and left inferior parietal lobe, which are regions often involved in unsuccessful encoding in younger adults. The implication of this finding is yet to be understood but it underscores a shift in the way older adults encode new information.

\section{Pathological Aging of Memory}

Although memory decline is considered a part of normal aging, it can also be a leading indicator of brain pathology, such as dementia of the Alzheimer's type. Alzheimer's disease is a progressive neurodegenerative disease characterized by loss of function and death of neurons in several brain areas. The medial-temporal cortex, particularly the entorhinal cortex, is vulnerable in the early stages of the disease, hallmarked by manifested deficits in episodic memory (Buckner 2004). It is the most common cause of dementia in pathological aging and symptoms advance to global compromise as the disease progresses, severely hampering life quality of patients and their caregivers. Considerable evidence suggests that individuals who will develop Alzheimer's disease exhibit cognitive deficits (including global cognitive ability, episodic memory, perceptual speed, and executive functioning) several years before a clinical diagnosis of dementia. Furthermore, pathology in the brain exists for at least a decade before clinical diagnosis of Alzheimer's disease is made (Gallagher and Koh 2011). From a clinical perspective, early identification of individuals at risk for developing Alzheimer's disease, combining genetic, biological, and cognitive markers, is imperative for maximizing effectiveness of interventions and is a research area currently under active investigation. 


\section{Cross-References}

- Individual Differences

- Memory: Autobiographical

- Memory: Training Methods and Benefits

> Plasticity of Aging

\section{References}

Barulli, D., \& Stern, Y. (2013). Efficiency, capacity, compensation, maintenance, plasticity: Emerging concepts in cognitive reserve. Trends in Cognitive Sciences, 17(10), 502-509.

Buckner, R. L. (2004). Memory and executive function in aging and AD: Multiple factors that cause decline and reserve factors that compensate. Neuron, 44(1), 195-208.

Chalfonte, B. L., \& Johnson, M. K. (1996). Feature memory and binding in young and older adults. Memory \& Cognition, 24(4), 403-416.

Craik, F. I. M. (1983). On the transfer of information from temporary to permanent memory. Philosophical Transactions of the Royal Society of London, B302, 341-359.

Gallagher, M., \& Koh, M. T. (2011). Episodic memory on the path to Alzheimer's disease. Current Opinion in Neurobiology, 21(6), 929-934.

Hertzog, C., Kramer, A. F., Wilson, R. S., \& Lindenberger, U. (2009). Enrichment effects on adult cognitive development: Can the functional capacity of older adults be preserved and enhanced? Psychological Science in the Public Interest, 9, 1-65.

Jacoby, L. L., \& Rhodes, M. G. (2006). False remembering in the aged. Current Directions in Psychological Science, 15(2), 49-53.

Kausler, D. H. (1994). Learning and memory in normal aging. New York: Academic.

Kempermann, G. (2008). The neurogenic reserve hypothesis: What is adult hippocampal neurogenesis good for? Trends in Neurosciences, 31(4), 163-169.

Light, L.L., Prull, M.W., La Voie, D., and Healy, M.R. (2000). Dual process theories of memory in old age.
In Models of Cognitive Aging (ed. T.J. Perfect and E. A. Maylor). New York, NY: Oxford University Press.

Lindenberger, U., \& Mayr, U. (2014). Cognitive aging: Is there a dark side to environmental support? Trends in Cognitive Sciences, 18(1), 7-15.

Lindenberger, U., von Oertzen, T., Ghisletta, P., \& Hertzog, C. (2011). Cross-sectional age variance extraction: What's change got to do with it. Psychology and Aging, 26(1), 34-47.

Maillet, D., \& Rajah, M. N. (2014). Age-related differences in brain activity in the subsequent memory paradigm: A meta-analysis. Neuroscience and Biobehavioral Reviews, 45, 246-157.

Nyberg, L., Lovden, M., Riklund, K., Lindenberger, U., \& Backman, L. (2012). Memory aging and brain maintenance. Trends in Cognitive Sciences, 16(5), 292-305.

Old, S. R., \& Naveh-Benjamin, M. (2008). Differential effects of age on item and associative measures of memory: A meta-analysis. Psychology and Aging, 23(1), 104-118.

Raz, N. (2005). The aging brain observed in vivo: Differential changes and their modifiers. In R. Cabeza, L. Nyberg, \& D. C. Park (Eds.), Cognitive neuroscience of aging: Linking cognitive and cerebral aging (pp. 19-57). New York: Oxford University Press.

Schacter, D. L., Koutstaal, W., \& Norman, K. A. (1997). False memories and aging. Trends in Cognitive Sciences, 1(6), 229-236.

Shing, Y. L., Werkle-Bergner, M., Brehmer, Y., Müller, V., Li, S.-C., \& Lindenberger, U. (2010). Episodic memory across the lifespan: The contributions of associative and strategic components. Neuroscience and Biobehavioral Reviews, 34, 1080-1091.

Smith, P. J., Blumenthal, J. A., Hoffman, B. M., Cooper, H., Strauman, T. A., Welsh-Bohmer, K., Browndyke, J. N., \& Sherwood, A. (2010). Aerobic exercise and neurocognitive performance: A meta-analytic review of randomized controlled trials. Psychosomatic Medicine, 72(3), 239-252.

Spencer, W. D., \& Raz, N. (1995). Differential effects of aging on memory for content and context: A metaanalysis. Psychology and Aging, 10(4), 527-539.

Yonelinas, A. P. (2002). The nature of recollection and familiarity: A review of 30 years of research. Journal of Memory and Language, 46(3), 441-517. 